\title{
DIAGNOSTICS FOR THE LCLS PHOTOINJECTOR BEAMLINE *
}

\author{
C. Limborg-Deprey ${ }^{\#}$, D.H. Dowell, J. Schmerge \\ SLAC, 2575 Sand Hill Road, Menlo Park, CA 94025, USA
}

\section{Abstract}

Two spectrometers have been added to the LCLS photoinjector beamline. The first one will be located close to the exit of the Photoinjector RF gun. With this diagnostic, we will measure beam energy, energy spread (correlated and uncorrelated), possibly deleterious structure in the longitudinal phase space induced by longitudinal space charge force, and slice thermal emittance ... This extensive characterization of the $5 \mathrm{MeV}$ electron bunch will be made possible by combining this spectrometer with other diagnostics (YAG screens and Cerenkov Radiator). A second spectrometer located at the end of the beamline has been designed to characterize the 6 dimensional phase space of the $135 \mathrm{MeV}$ beam to be injected in the main accelerator. At that second spectrometer station, we will measure energy, energy spread (correlated and uncorrelated), longitudinal phase space, slice emittances ... Those last two measurements require using this spectrometer in combination with the transverse RF deflecting cavity and with the quadrupole scan emittance station. The designs of these two spectrometers have been supported by simulations from MAD and PARMELA.

\section{INTRODUCTION}

The commissioning of the LCLS PhotoInjector beamline will start in January 2007. Diagnostics have been designed to reach the goal performances of $1 \mathrm{~mm}$.mrad emittance for a $1 \mathrm{nC}$ having a $100 \mathrm{~A}$ peak current. As presented in $[1,2]$, other operating points with lower charge will also be investigated. To achieve the optimum emittance compensation, a precise tuning of longitudinal parameters in the gun is required. Both the peak amplitude of the accelerating field, the injection phase needs to be known accurately. Another key component of the emittance compensation process is the solenoid position and calibration. A beam based alignment procedure similar to what was done at TTF [3] will be followed, but is not discussed here. In this paper, we discuss measurements aimed at reducing the ambiguity between the launch phase and the peak gun field. A description of the full $6 \mathrm{D}$ beam characterization at the end of the beamline before injection into the linac is given. The minimum emittance achievable in the Injector is determined by the uniformity of emission and cathode emittance. We accordingly start by discussing the key experiments to characterize emission.

A full layout of the beamline can be found in [4]. Figure 1 gives the locations of the diagnostics discussed in this paper.

\footnotetext{
*Work supported by supported by US Department of Energy, contract No. DE-AC02-76SF00515A06.

"limborg@slac.stanford.edu
}

\section{GUN EMISSION}

\section{Cathode Uniformity}

The cathode uniformity is measured by doing a pointto-point imaging of the cathode into the first (YAG1) or second screen (YAG2), by choosing the appropriate set of peak voltage, injection phase and solenoid current. This measurement requires the shortest pulse and lower charge to minimize aberrations due to $\mathrm{rf}$ and space charge fields.

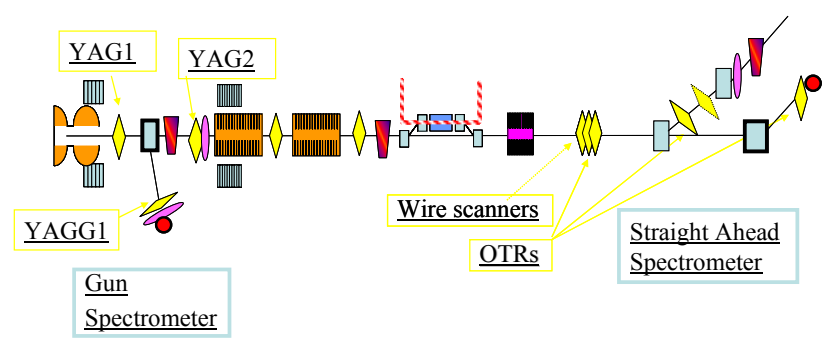

Figure 1: Layout of the diagnostics in the injector.

The drift after the Gun will be equipped with two YAG screens (YAG1, YAG2), Cerenkov Radiators for bunch length measurement, BPMs, two steering magnets and a current monitor.

\section{Cathode Emittance}

The cathode emittance is determined by measuring the rms beam size as a function of solenoid field at a YAG screen after the gun, as described in [5]. The method for optimizing the optics to improve the resolution of this measurement is described below.

The cathode emittance is the product of the divergence (or angular momentum) and beam size of the emitted electrons. Accordingly, we want to build an angle to point imaging system. The gun is a multiple lens system with 3 lenses corresponding to the exit/entrance/exit of the middle iris and exit iris of the 1.6 cell structure. Those irises act as lenses whose focal length depends on the phase and amplitude of the field. For our S-band gun, a $73 \mathrm{MV} / \mathrm{m}$ peak field and an injection phase of 32 degrees gives an optimal magnification on the YAG screen for a convenient range of solenoid fields. However, this parameter set is not unique. Figure 2.a shows the rms beam size as a function of solenoid current for different laser spot size radii. At the minimum, the image size is independent of the initial laser spot size. .This setting corresponds to angle-to-point imaging, and the Yag screen image shows the angular distribution of the beam at the cathode. For a zero thermal divergence or emittance, the minimum image size is indeed zero as shown in Figure 2.b.

In figure 3, we show that for different transverse momentum distribution the image on the screen has 
exactly the shape of the distribution for 3 cases of momentum distributions Gaussian, parabolic, and uniform.
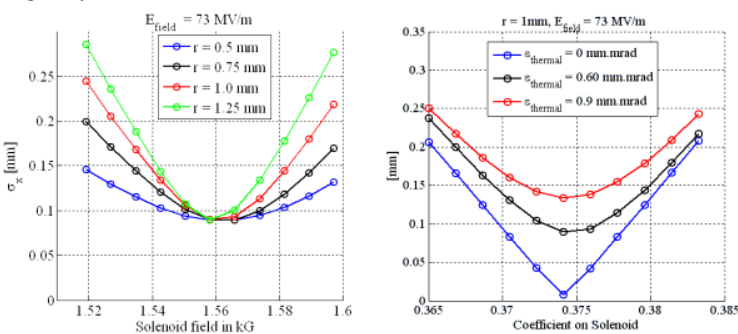

Figure 2: Beam Size at YAG1 as a function of solenoid strength (a) for $0.6 \mathrm{~mm} . \mathrm{mrad}$ per mm cathode emittance but various laser spot size (b) for different cathode emittance.

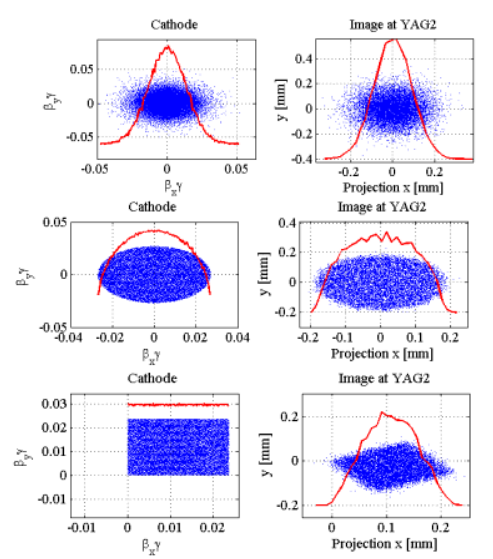

Figure 3: Left column: xy momentum distribution at cathode - Right column Image at second YAG screen.

The solenoid scans are accurate only if both the gun calibration and solenoid calibrations are well known. We show that the orthogonality of the voltage and phase of the gun can be uplifted from measurements based on the spectrometer.

\section{GUN SPECTROMETER}

The energy at the exit of the gun needs to be known to better than $0.1 \%$. Multiple experiments are described below which allow the distinction between an injection phase error and a voltage error.

\section{Design and Calibration}

The gun spectrometer was designed for point-to-point imaging of the YAG1 screen into a spectrometer screen located at $0.6 \mathrm{~m}$ from the exit of the spectrometer magnet. With this arrangement the beam is relay imaged from the cathode to the spectrometer screen. However, this configuration will only be useful at low charge, and quadrupoles have been added to control the beam size where space charge is important.

The spectrometer will be carefully calibrated in the magnetic measurements lab and its relative calibration will be assessed by comparing the steering of the beam onto YAGG1. As the solenoid rotates the beam through an angle proportional to the beam energy, the spectrometer energy calibration can be verified by measuring this rotation angle as a function of peak voltage. The spectrometer characteristics are given in Table 1.

Table 1: Gun Spectrometer parameters.

\begin{tabular}{l|l|l|l|l|}
\hline Length & Angle $\theta$ & Pole Face* & Dist. to screen \\
\hline $0.3 \mathrm{~m}$ & $84.46^{\circ}$ & $28.4^{\circ}$ & $0.617 \mathrm{~cm}$ \\
\hline
\end{tabular}

\section{Low Charge}

The curve of correlated energy spread as a function of injection phase has a minimum which varies with the peak voltage as shown in figure 4 . The spectrometer image is very linear with energy as shown in Figure4
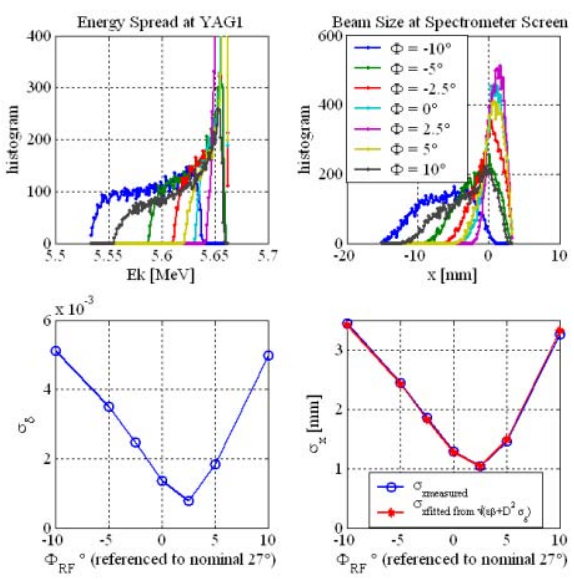

Figure 4: $1^{\text {st }}$ column Projection of energy spread at YAG1 $-2^{\text {nd }}$ column Horizontal profile at spectrometer screen.

The measurement of the minimum energy spread as a function of injection phase can be repeated for the high charge case. The phase offset introduced by the space charge limit is then known.

\section{Line Density Uniformity}

For a 57 degrees injection phase, the beam has a correlated energy spread of $0.45 \mathrm{MeV}$ at YAG1. This chirped beam will spread the longitudinal temporal profile across the bend-plane of the spectrometer screen. In the example showed in figure 5-6, a modulation of $+/-8 \%$ on top of the flat top beam had been artificially introduced. By adjusting the quadrupoles, the beam size was controlled given the large $0.45 \mathrm{MeV}$ correlated energy spread. One can see the microstructure in the horizontal projection with the correct periodicity. This measurement resolves the structure with a resolution of better than $0.5 \mathrm{ps}$, and accordingly better than a streak camera. This undesired longitudinal structure will be even better characterized at the second spectrometer. 


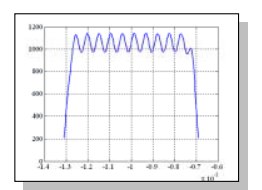

Figure 5: Initial laser distribution with $8 \%$ modulation.

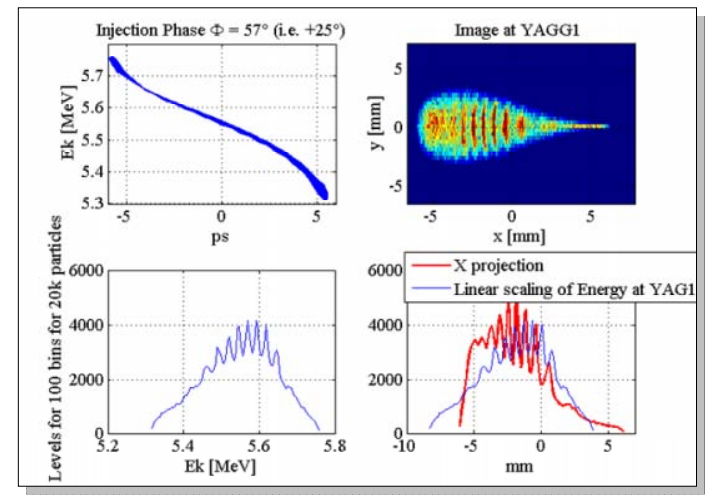

Figure 6: Shows the evolution of the beam with the laser profile presented in figure 5 (a) Longitudinal phase space at YAG1 location - (c) Projection of energy distribution at YAG1- (b) XY Image at spectrometer screen YAGG1- (d) Projection of horizontal distribution at YAGG1 (in the background same as (b).

\section{STRAIGHT AHEAD SPECTROMETER}

\section{Design}

Using the straight ahead spectrometer in conjunction with the transverse deflecting cavity [6], a direct measurement of the longitudinal phase space can be performed. The straight ahead spectrometer parameters were chosen so that the longitudinal phase space could be characterized with the nominal matching lattice used for injecting the $1 \mathrm{nC}$ bunch in the main linac. Therefore option to have the first dog leg magnet operate in pulsed mode to steal pulses is possible. The straight ahead spectrometer beamline contains an additional three quadrupoles after the spectrometer. They allow to obtain a large ratio between the Dispersion and the horizontal betatron function .

Table 2: Straight Ahead Spectrometer parameters.

\begin{tabular}{|l|l|l|l|}
\hline Length & Angle $\theta$ & Pole Face* & Dist. to screen \\
\hline $0.5 \mathrm{~m}$ & $35^{\circ}$ & $7.5^{\circ}$ & $2.3 \mathrm{~m}$ \\
\hline
\end{tabular}
* Pole Face rotation of both poles

\section{Longitudinal Phase Space Measurements}

By applying a $0.8 \mathrm{MV}$ on the transverse cavity [6], we generate a $+/-0.5 \mathrm{mrad}$ vertical kick along the $10 \mathrm{ps}$ long pulse. In figure 7 , the longitudinal phase space at the transverse deflecting cavity location (a) and the horizontal / vertical space at the spectrometer screen are given for various uncorrelated energy spreads.

We can extract a vertical slice to determine the energy spread. However, this requires the accurate knowledge of the local betatron functions. One can also use the horizontal projection. By varying the RF phase of the linac section, the maximum energy will slide along the bunch. Simulations, performed with PARMELA, show that we should be able to resolve better than $10 \mathrm{keV}$ with either method. The increase of uncorrelated energy spread from $3 \mathrm{keV}$ to $40 \mathrm{keV}$ produced by the laser heater will be easily measured at this station. By increasing incrementally the uncorrelated energy spread. The direct longitudinal phase space measurement will be compared with measurements performed using tomographic techniques.

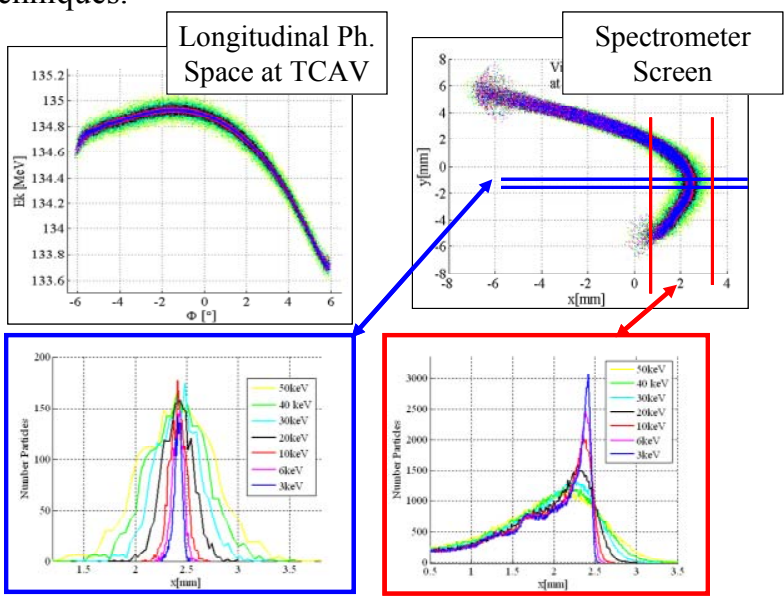

Figure 7: For nominal beam conditions in the transverse plane but with the uncorrelated energy spread increases from 0 to $50 \mathrm{keV}$ - (a) Longitudinal Phase Space at the transverse cavity location -(b) XY image at spectrometer screen- (c) Evolution of vertical slice -(d) Evolution of horizontal slice.

An horizontal slice emittance measurement will be available at the second OTR screen of the three screen emittance station. The time-vertical position correlation will be introduced by the transverse deflecting cavity, and the quadrupole (QE03) will be scanned.

Similarly a vertical slice emittance will be available by performing a quadrupole scan on a beam chirped from running the linac section off-crest and projected on the spectrometer screen. With those 3 types of emittnace measurements, we will have a full 6D emittance station at the end of our Injector beamline.

\section{REFERENCES}

[1] P.Emma et al;. "An Optimized low-charge configuration of the Linac Coherent Light Source", these proceedings, PAC 05.

[2] C.Limborg-Deprey et al. "Alternate tunings for the LCLS Photoinjector", these proceedings, PAC05.

[3] Beinhauer et al. "Beam Based Alignment of TTF RF Gun using V-Code", PAC 01.

[4] D.Dowell et al. "The Linac Coherent Light Source Photo-Injector Overview and Some Design Details", EPAC 04.

[5] W.Graves et al. "Measurement of Thermal Emittance for a copper PhotoCathode", PAC01.

[6] R.Akre et al. "A Transverse RF Deflecting Structure for bunch length and phase space Diagnostics." 